Brompton and Harefield NHS Foundation Trust, London, UK. ${ }^{\S}$ These two authors contributed equally to the study.

Correspondence: M.F. Moffatt, Molecular Genetics and Genomics Section, National Heart and Lung Institute, Dovehouse Street, London SW3 6LY, UK. E-mail: m.moffatt @imperial.ac.uk

Support Statement: The study was supported by the NIHR Respiratory Disease Biomedical Research Unit at the Royal Brompton and Harefield NHS Foundation Trust and Imperial College London, by a Research Excellence Award from Imperial College London, and by the Wellcome Trust (Grant number 077959/Z/05/Z).

Statement of Interest: None declared.

Acknowledgements: We are grateful to Michael Loebinger (Host Defence Unit, Royal Brompton and Harefield NHS Foundation Trust, London and Molecular Genetics and Genomics Section, National Heart and Lung Institute, Imperial College, London, UK) for his thoughtful comments on the paper.

\section{REFERENCES}

1 Staley JT, Konopka A. Measurement of in situ activities of nonphotosynthetic microorganisms in aquatic and terrestrial habitats. Annu Rev Microbiol 1985; 39: 321-346.
2 Turnbaugh PJ, Ley RE, Hamady M, et al. The human microbiome project. Nature 2007; 449: 804-810.

3 Rogers GB, Carroll MP, Serisier DJ, et al. Bacterial activity in cystic fibrosis lung infections. Respir Res 2005; 6: 49.

4 Cox MJ, Allgaier M, Taylor B, et al. Airway microbiota and pathogen abundance in age-stratified cystic fibrosis patients. PLoS One 2010; 5: e11044.

5 Saiman L, Siegel J. Infection control recommendations for patients with cystic fibrosis: microbiology, important pathogens, and infection control practices to prevent patient-to-patient transmission. Infect Control Hosp Epidemiol 2003; 24: S6-S52.

6 Hilty M, Burke C, Pedro H, et al. Disordered microbial communities in asthmatic airways. PLoS One 2010; 5: e8578.

7 Cole JR, Wang Q, Cardenas E, et al. The Ribosomal Database Project: improved alignments and new tools for rRNA analysis. Nucleic Acids Res 2009; 37: D141-D145.

8 Waine DJ, Honeybourne D, Smith EG, et al. Cross-sectional and longitudinal multilocus sequence typing of Pseudomonas aeruginosa in cystic fibrosis sputum samples. J Clin Microbiol 2009; 47: 3444-3448.

9 King PT. The pathophysiology of bronchiectasis. Int J Chron Obstruct Pulmon Dis 2009; 4: 411-419.

10 Rogers GB, Daniels TW, Tuck A, et al. Studying bacteria in respiratory specimens by using conventional and molecular microbiological approaches. BMC Pulm Med 2009; 9: 14.

11 Maughan $\mathrm{H}$, Cunningham KS, Wang PW, et al. Pulmonary bacterial communities in surgically resected noncystic fibrosis bronchiectasis lungs are similar to those in cystic fibrosis. Pulm Med 2012; 2012: 746358.

\title{
Exacerbation of respiratory symptoms in COPD patients may not be exacerbations of COPD
}

\section{To the Editor:}

Exacerbations of chronic obstructive pulmonary disease (COPD) are defined as acute events characterised by a worsening of the patient's respiratory symptoms, particularly dyspnoea, beyond day-to-day variation, leading to a change in medical treatment and/or hospitalisation [1, 2]. Exacerbations of COPD are a leading cause of hospitalisation and healthcare expenditures, particularly in frail, elderly patients. They alter the health-related quality of life and the natural course of disease, increasing the risk of mortality, both during and after the acute event $[1,2]$. Patients with COPD frequently have chronic comorbidities [1]. Several of these comorbidities may produce acute events, contributing to the increased morbidity and mortality in COPD exacerbations: acute myocardial infarction, congestive heart failure, cerebrovascular disease, cardiac arrhythmias and pulmonary circulation disorders [1].

By definition, acute exacerbations of COPD are considered respiratory diseases, with specific reference to the respiratory symptoms and to the organs involved (airways and lung). Indeed, respiratory viral or bacterial infections and air pollution are assumed to be the main causes of COPD exacerbations, but the exact contribution of infections is difficult to establish, and the aetiology of a large proportion of exacerbations remains undetermined $[1,3,4]$.

Although it is known that bronchoconstriction and hyperinflation contribute to the increase in dyspnoea in COPD patients, the chronic airway, pulmonary and systemic inflammation present in patients with stable COPD is associated with an acute transient inflammatory process when respiratory symptoms are exacerbated by infections and/or pollutants. Neutrophils and/or eosinophils increase in the airways and lung, together with inflammatory mediators and protein leakage (fig. 1). This acute "respiratory" inflammation is associated with systemic inflammation, as shown by the increase in circulating inflammatory cells and pro-inflammatory cytokines. These systemic effects represent an important pathogenic link between COPD and comorbidities, particularly cardiovascular diseases [3, 4]. Indeed, epidemics of influenza are associated with an increased risk of death, particularly in elderly patients with chronic diseases, and the cause of death may be both respiratory and cardiovascular, particularly in the case of myocardial infarctions and stroke [5]. 


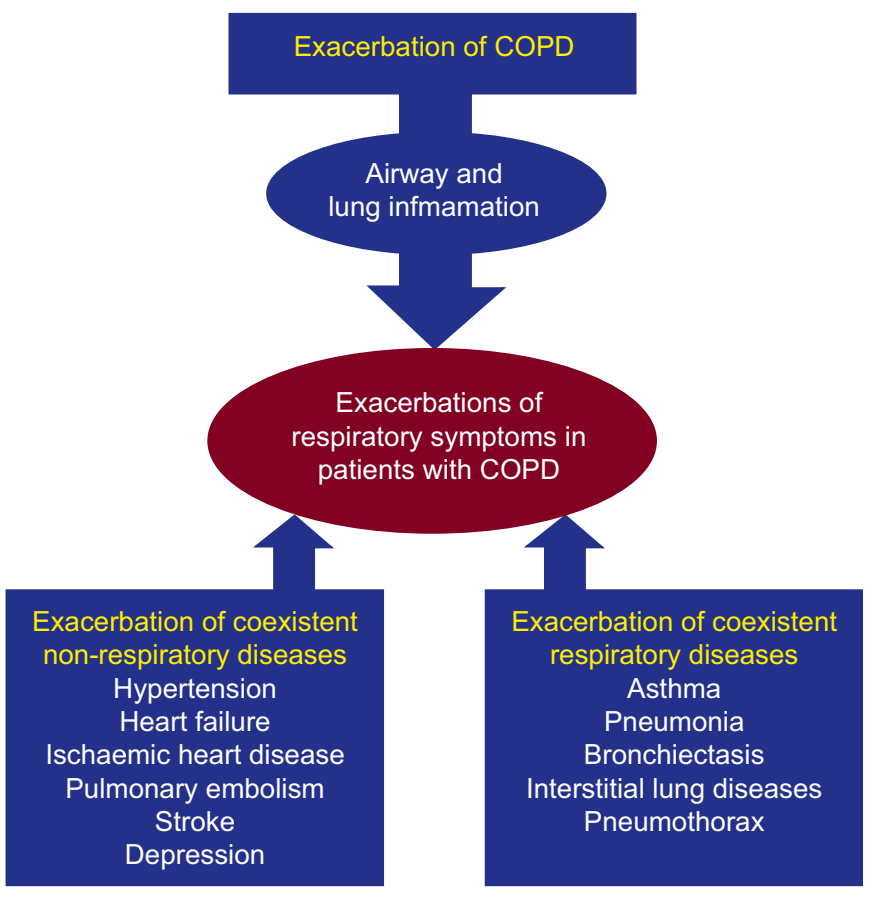

FIGURE 1. Exacerbation of respiratory symptoms in patients with chronic obstructive pulmonary disease (COPD) may be caused by respiratory infections and/or pollutants that cause acute inflammation of the airways and lung. These acute events may be correctly defined as exacerbations of COPD, but only after excluding other contributing mechanisms. Exacerbation of respiratory symptoms in patients with COPD and no comorbidities may be caused by acute independent events (e.g. pneumonia, pneumothorax, acute ischaemic heart disease and arrhythmias). These acute events should not be defined exacerbations of COPD, but rather exacerbations of respiratory symptoms in patients with COPD, followed by a description of the cause(s).

Some chronic diseases share with COPD not only the underlying chronic inflammation of the target organs (i.e. lungs, myocardium and vessels), but also some defining clinical manifestations, particularly fatigue and dyspnoea. In patients with multimorbidity, the exacerbation of respiratory symptoms may be particularly difficult to investigate. In fact, the exacerbation of respiratory symptoms in patients with COPD may be caused by acute inflammation of the airways and lung induced by infections and/or pollutants (in these cases the term exacerbation of COPD is probably appropriate), but even in these cases the airway and lung inflammation is associated with concomitant systemic inflammation that may affect other organs (e.g. vessels, heart and central nervous system) and thus decompensate concomitant cardiovascular, or metabolic diseases. Indeed, the exacerbations of respiratory symptoms caused by decompensation of these concomitant diseases in individual patients are sometimes difficult to distinguish from those primarily respiratory exacerbations of COPD (fig. 1).

In addition, exacerbations of respiratory symptoms in patients with COPD may also be caused by exacerbation of comorbidities (e.g. decompensated heart failure, arrhythmias and thromboembolisms), without involving the airways and lung (in these cases the term exacerbation of COPD is inappropriate and misleading). The clinical manifestations of decompensated heart failure, arrhythmias and ischaemic heart disease include dyspnoea and/or orthopnoea, cough or wheezing. Sometimes, electrocardiogram, echocardiogram and biomarkers such as $\mathrm{N}$ terminal pro-brain natriuretic peptide (NT-proBNP) and troponin may help to assess the specific contribution of each disorder to respiratory symptoms [6]. Pulmonary embolism may manifest as shortness of breath, cough and sputum, and imaging or blood Ddimers may be required to assess its contribution [7]. Finally, depression is a very frequent comorbidity of COPD, creating a vicious circle with dyspnoea, so that the presence of anxiety and depression is associated with early admission to hospital in the course of a COPD exacerbation because of more intense respiratory symptoms [3, 8]. Interestingly, abnormalities of biomarkers or imaging (e.g. NT-proBNP, high-sensitivity cardiac troponin $\mathrm{T}$, platelet function, or neutrophil gelatinase-associated lipocalin) may be present in patients with COPD exacerbations without overt clinical manifestations of myocardial damage and/ or dysfunction, pulmonary embolism, or renal failure. These biochemical abnormalities are associated with increased mortality [9]. Interestingly, exacerbations of respiratory symptoms may be caused by exacerbations of chronic respiratory diseases that may be present in patients with COPD, e.g. asthma, bronchiectasis and interstitial lung diseases. In these cases it would be almost impossible to identify the main cause or the relative contribution.

Finally, exacerbation of respiratory symptoms in patients with COPD and no recognised concomitant chronic diseases may also be caused by new acute events (e.g. myocardial infarction, arrhythmias, pneumonia and pneumothorax). The latter cases should also not be defined as COPD exacerbations.

The current definition of COPD exacerbation is only descriptive and does not characterise an exacerbation of COPD in itself. Rather, exacerbation of COPD is an exacerbation of the respiratory symptoms in patients with COPD that may be caused by other non-respiratory or respiratory mechanisms. This difference is not semantic, but rather clinically relevant! The complex pathogenic interactions between COPD and exacerbation of various concomitant respiratory and/or non-respiratory diseases results in degrees of summing, overlapping or mimicking of respiratory symptoms diagnosed as a COPD exacerbation.

For this reason we suggest that the term "exacerbation of COPD" be changed to the broader term, "exacerbation of respiratory symptoms in patients with COPD", with the aim to stimulate physicians to carefully investigate in the individual patient the complex respiratory and non-respiratory mechanisms potentially involved, and thus treat the patient more appropriately (fig. 1). Beyond this definition issue, it is imperative to continue the search for reliable biomarkers for aetiological diagnosis of exacerbations of respiratory symptoms in patients with COPD.

Similar to the above proposed change in terminology, we suggest that exacerbations of respiratory symptoms occurring in patients who have COPD and other concomitant chronic respiratory (e.g. asthma, bronchiectasis and pulmonary fibrosis) or non-respiratory (e.g. chronic heart failure, arrhythmias and obesity) diseases should be changed to exacerbations of respiratory symptoms in patients with multi-morbidity, including COPD and concomitant chronic diseases (e.g. asthma, and/or bronchiectasis, and/or chronic heart failure).

Considering the complexity of COPD in both the stable condition and during exacerbations of respiratory symptoms, 
COPD patients in general, but in particular COPD patients with comorbidities and exacerbations of respiratory symptoms, should be carefully monitored through a control panel for their complexity [10].

\section{Bianca Beghé*, Alessia Verduri*, Mihai Roca* and Leonardo M. Fabbri*}

*Section of Respiratory Diseases, Dept of Oncology Haematology and Respiratory Diseases, University of Modena and Reggio Emilia, Modena, Italy. "Faculty of Medicine, Dept of Pneumology, "Grigore T. Popa" University of Medicine and Pharmacy Iasi, Iasi, Romania.

Correspondence: L.M. Fabbri, Dept of Oncology Haematology and Respiratory Diseases, University of Modena and Reggio Emilia, Policlinico di Modena, Largo del Pozzo 71, 41124 Modena, Italy. E-mail: leonardo.fabbri@unimore.it

Statement of Interest: Conflict of interest information can be found alongside the online version of this article at www.erj. ersjournals.com

\section{REFERENCES}

1 GOLD. Global Strategy for the Diagnosis, Management, and Prevention of COPD, 2011. www.goldcopd.org/Guidelines/guide lines-resources.html Date last accessed: October 1, 2012.
2 Soriano JB, Brusasco V, Dinh-Xuan AT. The European Respiratory Journal makes COPD a priority. Eur Respir J 2011; 38: 999-1001.

3 Bafadhel M, McKenna S, Terry S, et al. Acute exacerbations of COPD: identification of biological clusters and their biomarkers. Am J Respir Crit Care Med 2011; 184: 662-671.

4 Mallia P, Message SD, Gielen V, et al. Experimental rhinovirus infection as a human model of chronic obstructive pulmonary disease exacerbation. Am J Respir Crit Care Med 2011; 183: 734-742.

5 Madjid M, Miller CC, Zarubaev VV, et al. Influenza epidemics and acute respiratory disease activity are associated with a surge in autopsy-confirmed coronary heart disease death: results from 8 years of autopsies in 34,892 subjects. Eur Heart J 2007; 28: 1205-1210.

6 Mogelvang R, Goetze JP, Schnohr P, et al. Discriminating between cardiac and pulmonary dysfunction in the general population with dyspnoea by plasma pro-B-type natriuretic peptide. J Am Coll Cardiol 2007; 50: 1694-1701.

7 Bertoletti L, Quenet S, Mismetti P, et al. RIETE Investigators. Clinical presentation and outcome of venous thromboembolism in COPD. Eur Respir J 2012; 39: 862-868.

8 Papaioannou AI, Bartziokas K, Tsikrika S, et al. The impact of depressive symptoms on recovery and outcome of hospitalised COPD exacerbations. Eur Respir J 2013; 41: 815-823.

9 Chang CL, Robinson SC, Mills GD, et al. Biochemical markers of cardiac dysfunction predict mortality in acute exacerbations of COPD. Thorax 2011; 66: 764-768.

10 Agusti A, Macnee W. The COPD control panel: towards personalised medicine in COPD. Thorax 2012; [in press DOI: 10.1136/thoraxjnl-2012-202772].

DOI: 10.1183/09031936.00180812

\section{Tracheal oxalosis associated with Aspergillus niger tracheobronchitis}

\section{To the Editor:}

Aspergillus is a widespread mould that can cause a variety of human diseases, usually in the setting of immunosuppression. Invasive pulmonary aspergillosis is the most aggressive form of Aspergillus infection and it is associated with morbidity and mortality. Invasive Aspergillus tracheobronchitis is a rare entity, primarily affecting lung transplant recipients, and patients with AIDS or chronic obstructive pulmonary disease (COPD). Aspergillus niger, and to a lesser extent $A$. fumigatus, can cause calcium oxalate crystals to accumulate in the lung, a condition termed pulmonary oxalosis. Tracheal oxalosis due to invasive Aspergillus tracheobronchitis has, to our knowledge, not been described before. Here, we report the case of a patient with probable invasive Aspergillus tracheobronchitis who developed tracheal oxalosis. Clinical, radiological and pathological correlation is given.

Invasive pulmonary aspergillosis is the most aggressive form of Aspergillus infection and it is related with a high morbidity and mortality [1]. The majority of patients with invasive aspergillosis are critically ill, requiring intensive care unit (ICU) admission. The presence of Aspergillus in the airways of critically ill patients carries higher mortality risk [2], and should prompt further diagnostic evaluation, including fibreoptic bronchoscopy [3].

Invasive Aspergillus tracheobronchitis is thought to be a rare entity, primarily affecting lung transplant recipients [4], patients with AIDS [5] and those with COPD [6]. Oxalosis is a condition in which calcium oxalate crystals accumulate in different organs. Oxalosis has been documented in patients with pulmonary aspergillosis [7] or mucormycosis [8] infections. However, there is no previous report in the medical literature of tracheal oxalosis associated with Aspergillus infection.

Here we present an immunosuppressed patient who developed probable invasive Aspergillus tracheobronchitis and the formation of calcium oxalate monohydrate crystals in the tracheal wall.

A 67-year-old female was assessed for fever and generalised myalgia. She had a medical history of splenectomy due to idiopathic thrombocytopenia. She was diagnosed with septic shock due to Streptoccocus pneumoniae infection and was admitted to the ICU. Chest radiograph on admission showed no infiltrates in the lung parenchyma. She rapidly developed multiple organ dysfunction syndrome with acute respiratory distress syndrome 